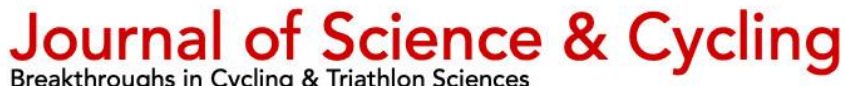

Original article

\section{Pacing strategy during Olympic Cross-Country Mountain Bike: effects of performance level, age and sex}

\author{
Everton Crivoi do Carmo ${ }^{1,2,3^{*}}$; Allan Inoue ; Cesar Cavinato Cal Abad ${ }^{1,2}$; Frederico Antonio \\ dos Santos Andrade ${ }^{5}$; Valmor Tricoli; Renato Barroso ${ }^{6}$ \\ ${ }^{1}$ Department of Physical Education, Senac University Center, São Paulo, SP, Brazil \\ ${ }^{2}$ School of Physical Education and Sport - University of São Paulo, São Paulo, SP, Brazil. \\ ${ }^{3}$ Nucleus of High Performance in Sports - NARSP, São Paulo, SP, Brazil. \\ ${ }^{4}$ Brazilian Navy, CEFAN, Research Laboratory of Exercise Science, Rio de Janeiro, RJ, Brazil \\ ${ }^{5}$ Singularidades Institute - iS, São Paulo, SP, Brazil. \\ ${ }^{6}$ Department of Sport Sciences, Faculty of Physical Education - State University of Campinas, \\ Campinas, SP, Brazil
}

* Correspondence: Everton Crivoi do Carmo. everton.ccarmo@sp.senac.br

Received: 21 February 2019; Accepted: 8 December 2019; Published: 30 June 2020

\begin{abstract}
Objectives: the aim of the present study was to verify the pacing strategy during a season of a Cross-Country Mountain Bike (XCO-MTB) and the effects of performance level, age and sex. Method: Overall, 802 paces in 4 age-sex categories were analyzed: male elite (EliteM; $\mathrm{n}$ $=272)$, female elite (EliteF; $n=170)$, male under-23 (U23M; $n=247)$ and female under-23 (U23F; $\mathrm{n}=113$ ). Races were divided into Initial Lap; middle one (Middle1); middle two (Middle2) and Final Lap. The athletes were divided into high performance (HP); intermediated performance (IP) and low performance (LP). The magnitude-based inference and the effect size were assessed to check the changes clinically important. Results: it was observed a similar fast-start strategy for all categories. Both HP EliteM and EliteF athletes showed higher speed in Final Lap than LP (EliteM - ES $=0.5 ; 90 \% \mathrm{CI}-0.8$ to -0.2 ; very likely and EliteF $-\mathrm{ES}=1.0 ; 90 \% \mathrm{CI}-1.4$ to -0.6 ; almost certain). The U23F athletes showed higher speeds in Initial Lap than EliteF (ES $=0.21 ; 90 \% \mathrm{CI}$ 0.1 to 0.5 ; likely), however in Final Lap the speeds was lower in U23F (ES $=1 ; 90 \% \mathrm{CI}-1.3$ to -0.6 ; very likely). Conclusion: the fast-start strategy is typically used during a XCO-MTB race independently of performance level, age or sex. HP Elite athletes are able to maintain higher speeds in the Final Lap. U23F athletes used to do a more variable pacing strategy with more aggressive fast-start strategy and lower speed in Final Lap than EliteF.
\end{abstract}

Keywords: cycling, endurance, performance, training

\section{Introduction}

The pacing strategy is an essential component of endurance performance (Abbiss and Laursen, 2008). During a Cross-Country Mountain Bike (XCO-MTB) race the pacing strategy is characterized by a fast-start, followed by an even pacing (Abbiss et al., 2013). The initial high speed seems to be a tactical decision (Impellizzeri and Marcora, 2007) since a significant and positive association has been observed between the position at the beginning of the race and the race outcome (Macdermid and Morton, 2012). These authors reported that it is unlikely for competitors to finish the race high ranked if they start in the back positions.

However, this pacing profile may be influenced by factors as circuit characteristics (Abbiss et al., 2013; Martin et al., 2012), competitive level (Konings, Schoenmakers, Walker and Hettinga, 
2016; Tomazini et al., 2015), sex and age (Abbiss and Laursen, 2008; Deaner and Lowen, 2016).

In the first part of a race, athletes of different competitive levels showed similar relative speeds (Konings et al., 2016; Renfree, Crivoi do Carmo, Martin, and Peters, 2015). However, when compared top and bottom athletes different overall pacing strategies have been described during Cyclo-Cross (Bossi, O'Grady, Ebreo, Passfield, and Hopker, 2018) and XCOMTB races (Abbiss et al., 2013). Abbiss et al. (2013) reported that the best classified athletes performed a more constant pacing strategy compared to the worst classified athletes who reduced their speed in the second half of a XCOMTB race. Therefore, high performance athletes seems to be able to maintain higher speeds during the remaining race distance compared to those with worse performance. These differences could occur due the athletes' ability to maintain high intensity during the race (de Koning et al., 2011; Esteve-Lanao, LarumbeZabala, Dabab, Alcocer-Gamboa, and Ahumada, 2014; Renfree and St Clair Gibson, 2013; Tucker, 2009).

Sex and age also seems to have important influence on pacing strategy. However, few studies were carried out comparing pacing strategy by sex or age. One of them observed that women had less variation in speed compared to men during a marathon running (March, Vanderburgh, Titlebaum, and Hoops, 2011). During cycle-cross races men and women seem to distribute the exercise intensity differently. Variations in speed during the race are smaller and normalized speeds (lap speed expressed as percent of average race speed) in the final lap are higher in women events (Bossi et al., 2018). Regarding age, differences in pacing strategy were observed when children of different ages (5-6 to 14) are compared. Younger children exhibited a negative pacing strategy, demonstrating an inability to anticipate the demands of the efforts, whereas older children presented a more conservative U-shaped strategy (Micklewright et al., 2012). Specifically in XCO-MTB, a more variable pacing strategy during 2009 World Championship, verified by standard deviation of the laps, was observed in junior male athletes compared to elite male athletes (Abbiss et al., 2013), indicating an effect of age on pacing strategy.

Differences in pacing strategies in young athletes could be associated to a decisionmaking process and experience during endurance sports (Elferink-Gemser and Hettinga, 2017). Additionally, the more variable pacing strategy observed in woman and junior male athletes (Abbiss et al., 2013) have been suggested to be a consequence of more time spent during technical ascents or during high speeds parts of the course. Therefore, the terrain characteristics may affect pacing strategy and variable gradient course is an important challenge to adjust the pacing strategy throughout the race (Cangley, Passfield, Carter, and Bailey, 2011; Martin et al., 2012). In this context, it is likely that pacing strategy analysis in a single XCO-MTB race should not be extrapolated to others races during a season and a broader analysis are necessary.

A competitive season during the calendar have two official (XCO-MTB) competitions organized by the Union Cycliste Internationale (UCI): the World Championships, which is played in a single event, and the XCO-MTB World Cup, which includes six races held between May and August. These competitions are performed in a closed circuit and the courses can vary in both length (between four and six kilometers) and lap numbers (between six and eight laps) (Costa and Oliveira, 2010). Courses are also characterized by different types of terrain such as dirt roads, rock gardens, climbs, technical descents, and single tracks (Impellizzeri, Sassi, RodriguezAlonso, Mognoni, and Marcora, 2002). Therefore, a better understanding about the strategy adopted during an entire season is necessary to help coaches and sport scientists during the preparation process of their athletes. Given the potential influence of terrains characteristics, level of athlete's performance, sex and age on pacing strategy, the main purpose of the present study was to characterize 
pacing strategy during an entire competitive season of XCO-MTB and to verify if performance level, sex and age would have some influence on the strategy adopted.

\section{Methods}

The present study has a descriptive, observational and retrospective design. Results of the six stages of the 2015 XCO-MTB World Cup (Czech Republic, Germany, Switzerland, Canada, United States of America and Italy) and the World Championships (Vallnord, Andorra) were considered for analysis. The data was accessed throughout the public domain website available at http://www.uci.ch/mountainbike/results/.

The final results and each lap in male Elite (EliteM), female Elite (EliteF), male under-23 (U23M) and female under-23 (U23F) were analyzed with a total of 802 pacing strategies (272 for EliteM, 170 for EliteF, 247 for U23M and 113 for U23F). The total distance, number of laps,

Table 1. Total distance, number of laps, number of athletes and athletes who completed each race of the uci world cup and the world championships.

\begin{tabular}{|c|c|c|c|c|c|}
\hline Races & Category & $\begin{array}{c}\text { Total distance } \\
(\mathbf{k m})\end{array}$ & Laps & Athletes (n) & $\begin{array}{l}\text { Concluding } \\
\text { athletes (n) }\end{array}$ \\
\hline Nove Mesto & EliteM & 24.6 & 6 & 87 & 52 \\
\hline \multirow[t]{4}{*}{ (Czech Republic) } & EliteF & 20.5 & 5 & 61 & 37 \\
\hline & U23M & 20.5 & 5 & 99 & 59 \\
\hline & U23F & 16.4 & 4 & 48 & 29 \\
\hline & EliteM & 33.6 & 8 & 52 & 31 \\
\hline Albstadt & EliteF & 29.4 & 7 & 38 & 23 \\
\hline \multirow[t]{2}{*}{ (Germany) } & U23M & 29.4 & 7 & 51 & 31 \\
\hline & U23F & 25.2 & 6 & 27 & 16 \\
\hline & EliteM & 33.2 & 8 & 58 & 35 \\
\hline Lenzerheide & EliteF & 29.05 & 7 & 33 & 20 \\
\hline \multirow[t]{2}{*}{ (Switzerland) } & U23M & 29.05 & 7 & 56 & 34 \\
\hline & U23F & 24.9 & 6 & 22 & 13 \\
\hline Mont-Sainte-Anne & EliteM & 27.3 & 7 & 60 & 36 \\
\hline \multirow[t]{3}{*}{ (Canada) } & EliteF & 23.4 & 6 & 33 & 20 \\
\hline & U23M & 23.4 & 6 & 37 & 22 \\
\hline & U23F & 19.5 & 5 & 19 & 11 \\
\hline Windham & EliteM & 29.4 & 7 & 60 & 36 \\
\hline \multirow[t]{3}{*}{ (USA) } & EliteF & 25.2 & 6 & 38 & 23 \\
\hline & U23M & 25.2 & 6 & 40 & 24 \\
\hline & U23F & 21 & 5 & 15 & 9 \\
\hline Val di Sole & EliteM & 28.7 & 7 & 73 & 44 \\
\hline \multirow[t]{3}{*}{ (Italy) } & EliteF & 24.6 & 6 & 42 & 25 \\
\hline & U23M & 24.6 & 6 & 73 & 44 \\
\hline & U23F & 20.5 & 5 & 28 & 17 \\
\hline Vallnord & EliteM & 25.2 & 6 & 63 & 38 \\
\hline \multirow[t]{3}{*}{ (Androrra) } & EliteF & 25.2 & 6 & 38 & 23 \\
\hline & U23M & 21 & 5 & 55 & 33 \\
\hline & U23F & 21 & 5 & 30 & 18 \\
\hline
\end{tabular}


number of athletes and athletes who completed each race of the UCI World Cup and the World Championships are described in Table 1.

$\mathrm{M}=$ male; $\mathrm{F}=$ female; $\mathrm{U} 23$ = athletes under 23 years old.

The average speed $(\mathrm{km} \cdot \mathrm{h}-1)$ of each lap was normalized by the average speed of the race and expressed as percent values (Renfree, Carmo, and Martin, 2016). Since the number of laps varies according to the race circuit, sex and age, to compare the entire season and the different categories each race was divided into four stages: Initial Lap - the first lap of the race; first half of the race (Middle1) - from the second lap up to the lap corresponding to half of the race (i.e. in a race with 6 laps, the second and third laps); second half of the race (Middle2) - from the lap corresponding to half of the race up to the second last lap; and Final Lap - the last lap of the race. This division allowed identifying the speed distribution pattern by steps throughout the season and the comparison of this pattern between sex and age.

To investigate the influence of the performance level on the pacing strategy, athletes who finished the race were subdivided and classified into quintiles as follow: first quintile (top $20 \%$ athletes) $=$ high performance $(\mathrm{HP})$; third quintile $=$ intermediated performance (IP); and fifth quintile $=$ low performance (LP). Athletes who were not classified into any of the groups were excluded from the analysis. This classification allowed the differentiation in performance levels, making it possible to identify differences in pacing strategy (Abbiss et al., 2013). For comparisons between sex and age just the HP group was analyzed.

\section{Statistical Analysis}

The normality of data was tested using the Shapiro-Wilk test. The data are expressed as mean \pm standard deviation. The magnitude of the differences was assessed using the effect size (ES) analysis and qualitatively interpreted by using values of $0.2,0.6,1.2,2.0$, and 4.0 of the variation as thresholds for small, moderate, large, very large, and extremely large differences (Hopkins, Marshall, Batterham, and Hanin, 2009). Additionally, to check the minimal important change, the smallest worthwhile change (SWC) was assessed $(0.2 \times$ standard deviation). The quantitative chances of finding differences were assessed qualitatively as follows: $<1 \%$, almost certainly not; $1 \% 5 \%$, very unlikely; $5 \% 25 \%$, unlikely; $25 \% 75 \%$, possible; $75 \% 95 \%$, likely; $95 \% 99 \%$, very likely; $>99 \%$, almost certain. If the chances of having better and poorer results were both $>5 \%$, the true difference was rated as unclear (Hopkins, Marshall, Batterham, and Hanin, 2009).

\section{Results}

\section{Pacing strategy}

Overall it was observed a similar pacing strategy for all categories (FIGURE 1). In EliteM the Initial Lap was performed on higher relative speed than Middle1 (106.2\% vs $99.6 \%$; MD = $6.6 \%$; $\mathrm{ES}=0.9 ; 90 \% \mathrm{CI} 1.2$ to $0.6 ; 99.9 / 0.01 / 0.0-$ almost certain), Middle2 (98.1\%; MD = 8.2\%; ES $=1.1 ; 90 \%$ CI 1.4 to $0.8 ; 99.9 / 0.01 / 0.0-$ almost certain), and Final Lap (99.1\%; MD = 7.1\%; ES = $0.9 ; 90 \%$ CI 1.3 to $0.6 ; 99.9 / 0.01 / 0.0$ - almost certain). The relative speed in Middle1 was higher than Middle2 (MD $=1.5 \%$; ES = 0.8; $90 \%$ CI 1.1 to $0.4 ; 99.6 / 0.4 / 0.0$ - almost certain). However, it was observed a possible trivial effect between Final Lap and Middle1 (MD = $0.4 \%$; ES $=0.2 ; 90 \% \mathrm{CI} 0.4$ to $-0.08 ; 40 / 60 / 0$ ) and an unclear effect between Final Lap and Middle2 $(\mathrm{MD}=1.1 \%$; $\mathrm{ES}=0.4 ; 90 \% \mathrm{CI} 0.13$ to 0.7 ; $52.6 / 4.6 / 42.6)$.

In EliteF the relative speed of Initial Lap was almost certain higher compared to Middle1 $(105.8 \%$ vs $99.7 \%$; $\mathrm{MD}=6 \%$; ES $=0.9 ; 90 \%$ CI 1.3 to $0.6 ; 99.9 / 0.1 / 0.0)$, Middle2 $(98.0 \%$; $\mathrm{MD}=$ $7.8 \%$; ES $=1.2 ; 90 \%$ CI 1.6 to $0.8 ; 99.9 / 0.01 / 0.0$ ), and Final Lap $(100.0 \%$; MD $=5.8 \%$; ES $=0.9$; $90 \%$ CI 1.2 to $0.5 ; 99.9 / 0.1 / 0.0)$. The relative speed in Middle1 was almost certain higher than Middle2 (MD = 1.7\%; ES = 1; 90\%CI 1.3 to 0.6; 99.7/0.3/0.0). However, the effect was almost certain trivial between Final Lap and Middle1 $(\mathrm{MD}=0.2 \% ; \mathrm{ES}=0.1 ; 90 \% \mathrm{CI} 0.2$ to -0.4 ; 
0.8/99.2/0.0) and unclear between Final Lap and Middle2 (MD $=2 \%$; ES $=0.8 ; 90 \% \mathrm{CI} 0.4$ to $1.2 ; 53.7 / 2.5 / 43.7)$.

In U23M the relative speed in Initial Lap was almost certain higher than Middle1 $(107.1 \%$ vs $99.03 \% ; \mathrm{MD}=8.1 \%$; ES $=1.1 ; 90 \% \mathrm{CI} 1.4$ to 0.7 ; 99.9/0.01/0.0), Middle2 (98.04\%; MD = 9\%; ES = $1.2 ; 90 \%$ CI 1.5 to $0.8 ; 99.9 / 0.1 / 0.0$ ), and Final Lap $(98.4 \% ; \mathrm{MD}=8.7 \% ; \mathrm{ES}=1.1 ; 90 \% \mathrm{CI} 1.5$ to $0.7 ;$ $99.9 / 0.01 / 0.0)$. The effect was unclear between Middle1 and Middle2 (MD $=1 \%$; ES $=0.4 ; 90 \% \mathrm{CI}$ 0.7 to $0.1 ; 52.5 / 5.0 / 42.5)$, between Middle1 and Final Lap $(\mathrm{MD}=0.6 \%$; ES $=0.2 ; 90 \% \mathrm{CI} 0.5$ to $0.1 ; 50.9 / 8.1 / 40.9)$ and between Middle2 and Final Lap (MD $=0.4 \%$; ES $=0.1 ; 90 \%$ CI 0.18 to $0.5 ; 48.5 / 12.6 / 38.7)$.

Finally, the relative speed in U23F was almost certain higher for Initial Lap than Middle1 (107.7\% vs $99.2 \%$; $\mathrm{MD}=8.5 \%$; $\mathrm{ES}=1.2 ; 90 \% \mathrm{CI}$
1.5 to $0.8 ; 99.9 / 0.01 / 0.0)$, Middle2 (97.1\%; $\mathrm{MD}=$ $10.6 \%$; ES $=1.5 ; 90 \%$ CI 1.8 to $1.09 ; 99.9 / 0.01 / 0.0$ ), and Final Lap (97.1\%; MD = 10.6\%; ES = 1.5; $90 \%$ CI 1.8 to $1.1 ; 99.9 / 0.01 / 0.0)$. The relative speed was likely higher in Middle1 than Middle2 $(\mathrm{MD}=2.1 \%$; ES $=0.7 ; 90 \% \mathrm{CI} 1.09$ to 0.39; 87.7/0.1/3.1) and Final Lap (MD = 2.1\%; ES $=0.8 ; 90 \%$ CI 1.15 to $0.45 ; 87.7 / 0.1 / 3.1-$ likely). However, it was an almost certain trivial effect between Middle2 and Final Lap (MD $=0.1 \%$; ES $=0.1 ; 90 \% \mathrm{CI} 0.3$ to $-0.3 ; 0.0 / 100 / 0.0)$.

\section{Pacing strategy by performance level}

The differences in relative average speed according to the performance level can be observed in Figure 2.
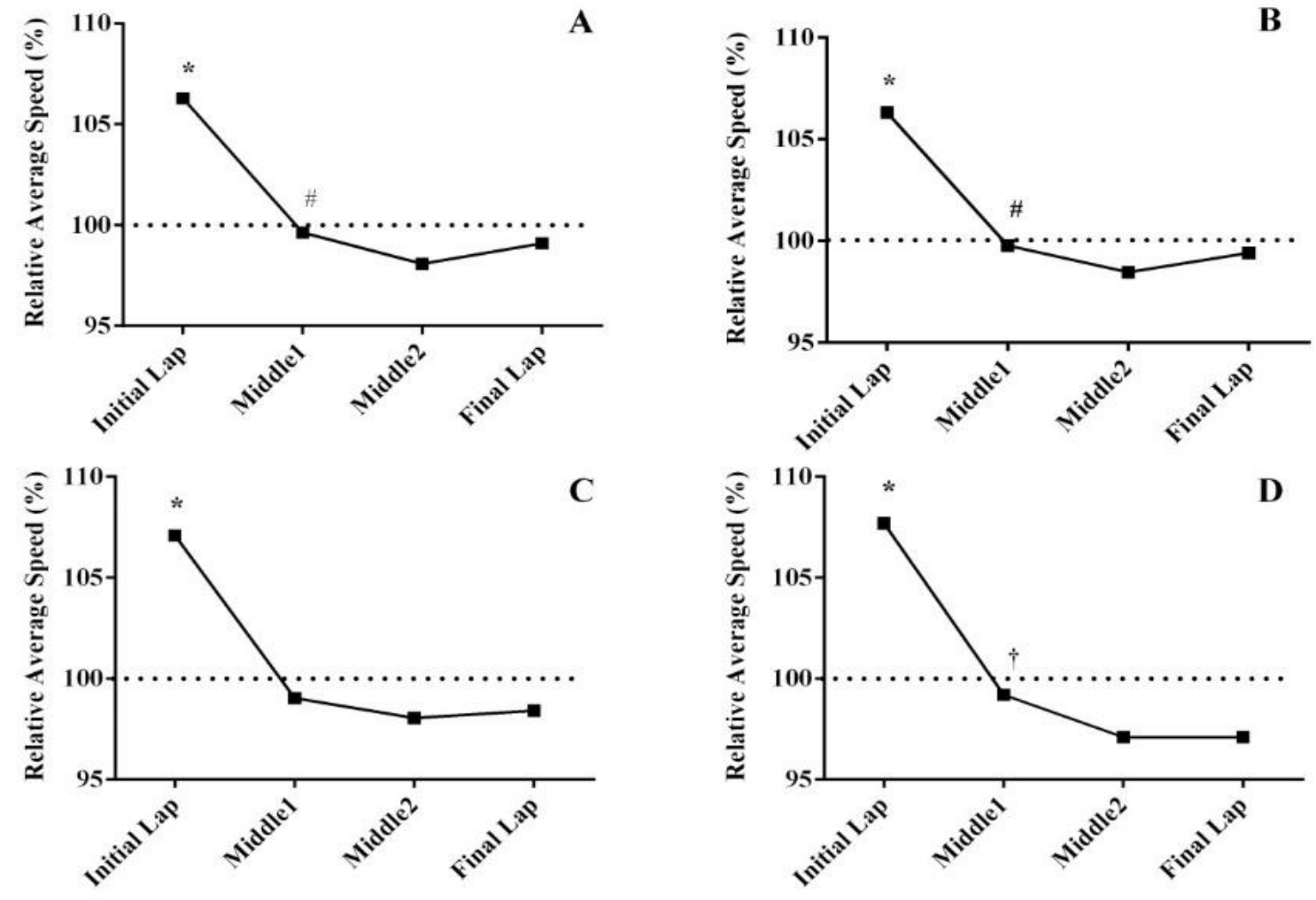

Figure 1. Overall pacing strategy by categories. $A=$ EliteM; $B=$ EliteF; $C=U 23 M ; D=U 23 F$.

Dotted line represents the race average speed. The standard deviation was removed for better visualization. *almost certain effect compared to M1, M2 and FL; \# almost certain effect compared to M2; + likely effect comparing to M2 and FL. 


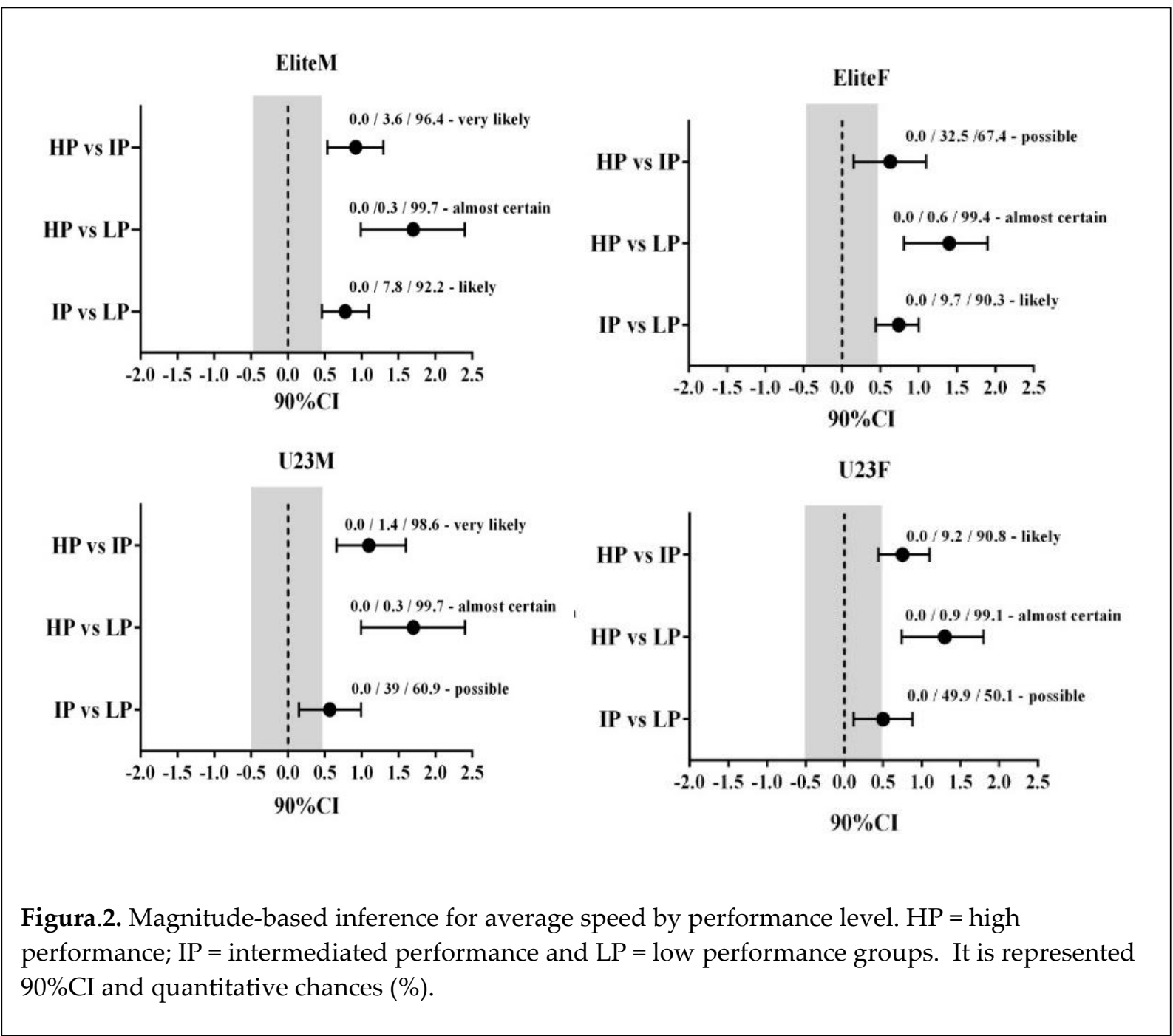

The pacing strategy by performance level can be observed in Figure 3. It was observed unclear effects in EliteM in Initial Lap $(\mathrm{HP}=106.2 \pm$ $10.1 \% ; \mathrm{IP}=105.6 \pm 8.8 \% ; \mathrm{LP}=105 \% \pm 8.4 \%)$, Middle1 (HP $=99.6 \pm 1.7 \%$; IP $=100 \pm 1.7 \%$; LP $=100.8 \pm 1.8 \%)$ and Middle2 $(\mathrm{HP}=98.1 \pm 2.2 \%$; $\mathrm{IP}=98.2 \pm 1.9 \%$; LP $=98.1 \pm 2.2 \%)$. An unclear effect was also observed in Final Lap between HP $(99.1 \pm 2.7 \%)$ and IP $(97 \pm 4.4 \%)$. However, it was observed very likely higher relative speed in Final Lap when HP and LP were compared $(97 \pm 4.4 \% ; \mathrm{MD}=2.1 \%$; $\mathrm{ES}=0.5 ; 90 \% \mathrm{CI}-0.8$ to $0.2 ; 98.4 / 1.6 / 0.0)$.

Similarly, likely higher relative speed in Final Lap was found between IP and LP (MD $=1.4 \%$; $\mathrm{ES}=0.4 ; 90 \% \mathrm{CI}-0.6$ to $-0.08 ; 89.7 / 9.9 / 0.0)$.

The EliteF showed unclear effects between performance level in Initial Lap (HP = $105.8 \pm$
8.7\%; IP = $105.7 \pm 8.7 \% ; \mathrm{LP}=106.5 \pm 9.1 \%)$, Middle1 $(\mathrm{HP}=99.7 \pm 1.5 \%$; IP $=99.9 \pm 1.8 \%$; LP $=100.4 \pm 1.8 \%)$ and Middle $2(\mathrm{HP}=98 \pm 1.8 \%$; IP $=98.1 \pm 1.9 \%$; $\mathrm{LP}=97.7 \pm 2.2 \%)$. However, in Final Lap it was observed likely higher relative speed for HP than IP (HP $=100 \pm 2.8 \%$ vs $\mathrm{LP}=$ $98.2 \pm 2.7 ; \mathrm{MD}=1.7 \% ; \mathrm{ES}=0.6 ; 90 \% \mathrm{CI}-1.02$ to $0.27 ; 93.1 / 6.5 / 0.4)$ and almost certain higher relative speed for HP than LP (LP $=96.9 \pm 3.1 \%$; $\mathrm{MD}=3.1 \% ; \mathrm{ES}=1.1 ; 90 \% \mathrm{CI}-1.4$ to -0.6 ; $99.7 / 0.3 / 0.0)$. Finally, likely higher relative speed was found for IP than LP (MD $=1.3 \%$; ES $=0.4 ; 90 \%$ CI -0.8 to $-0.07 ; 82 / 15.9 / 2.1)$.

In U23M it was observed likely higher relative speed in Initial Lap to HP than LP $(107 \pm 10.2 \%$ vs $104.9 \pm 8.3 \% ; \mathrm{MD}=2.1 \%$; ES $=0.2 ; 90 \%$ CI 0.5 to $-0.1 ; 96.2 / 3.6 / 0.2)$. It was observed unclear effects in Middle1 $(\mathrm{HP}=99 \pm 2.2 \%$; IP $=99.1 \pm$ $1.9 \%$; $\mathrm{LP}=99.9 \pm 1.7 \%)$, Middle2 $(\mathrm{HP}=98 \pm$ $2.3 \% ; \mathrm{IP}=98.1 \pm 2.1 \% ; \mathrm{LP}=98.3 \pm 2.5 \%)$ and 
Final Lap $(\mathrm{HP}=98.4 \pm 2.9 ; \mathrm{IP}=99.1 \pm 3.6 ; \mathrm{LP}=$ $97.9 \pm 5.6 \%)$. In U23F there were observed unclear effects between performance level in Initial Lap $(\mathrm{HP}=107.8 \pm 9.6 \% ; \mathrm{IP}=108.1 \pm 9.9 \%$; $\mathrm{LP}=107.8 \pm 8.8 \%)$, Middle1 (HP $=99.3 \pm 2.4 \%$; $\mathrm{IP}=99.2 \pm 2.8 \% ; \mathrm{LP}=99.6 \pm 2.5 \%)$, Middle2 (HP $=97.1 \pm 3.2 \% ; \mathrm{IP}=97.3 \pm 2.6 \% ; \mathrm{LP}=96.6 \pm 2.9 \%$ ) and Final Lap $(\mathrm{HP}=97.1 \pm 2.8 \% ; \mathrm{IP}=96.9 \pm 3.3 \%$; $\mathrm{LP}=97.2 \pm 3.2 \%)$.

\section{Pacing strategy by sex and age}

Regarding sex, there were no differences in relative speed in Initial Lap when male and female Elite athletes were analyzed (106.3 \pm $10.1 \%$ vs $105.8 \pm 8.7 \%)$, Middle $1(99.6 \% \pm 1.7 \%$ vs $99.8 \pm 1.6 \%)$, Middle2 $(98.1 \pm 2.2 \%$ vs $98 \pm 1.9)$ and Final Lap (99.2 $\pm 2.8 \%$ vs $100 \pm 2.8)$.
In relation to age, there were no differences between EliteM and U23M in Initial Lap (106.3 \pm $10.1 \%$ vs $107.1 \pm 10.2 \%)$, Middle1 (99.6 $\pm 1.7 \%$ vs $99 \pm 2.2)$, Middle $2(98.1 \pm 2.2 \%$ vs $98 \pm 2.3 \%)$ and Final Lap $(99.2 \pm 2.8 \%$ vs $98.4 \pm 2.9 \%)$. It was observed likely lower relative speed in Initial Lap to EliteF compared to U23F (105.8 $\pm 8.7 \%$ vs $107.8 \pm 9.6 \% ; \mathrm{MD}=1.9 \%$; $\mathrm{ES}=0.3 ; 90 \% \mathrm{CI} 0.06$ to $0.55 ; 94.7 / 4.9 / 0.3)$. On the other hand, an almost certain higher speed was observed in Final Lap for EliteF ( $100 \pm 2.8 \%$ vs $97.1 \pm 2.9 \%$; $\mathrm{MD}=2.9 \%$; $\mathrm{ES}=-1 ; 90 \% \mathrm{CI}-1.3$ to $-0.6 ; 99.5 / 0.5 / 0.0)$. Unclear effects were observed for Middle1 (99.8 $\pm 1.9 \%$ vs $99.3 \pm 2.4 \%$ ) and Middle2 ( $98 \pm 1.9 \%$ vs $97.1 \pm$ $3.3 \%$ ). The $90 \% \mathrm{CI}$ and the harmful, trivial or beneficial effect to relative speed by sex and age can be observed in Figure 4 .
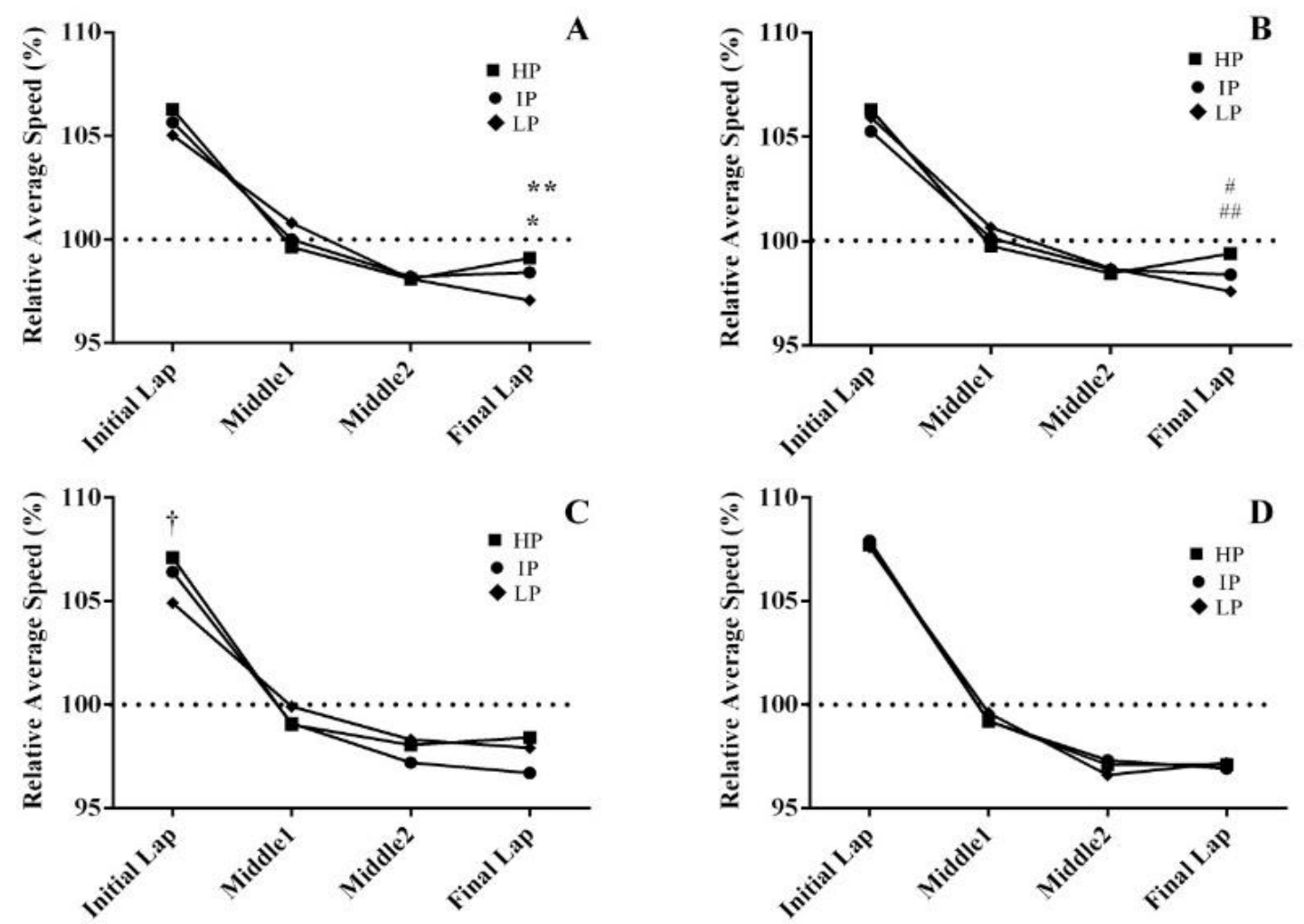

Figure 3. Pacing strategy by performance level. $A=$ EliteM; $B=E l i t e F ; C=U 23 M ; D=U 23 F . ~ H P=$ high performance; IP = intermediate performance; LP = low performance. Dotted line represents the average speed. The standard deviation was removed for better visualization. ${ }^{* *}$ very likely effect HP vs LP; *likely effect IP vs LP; \#likely effect HP vs IP; \#\#almost certain effect HP vs LP; † likely effect HP vs LP. 

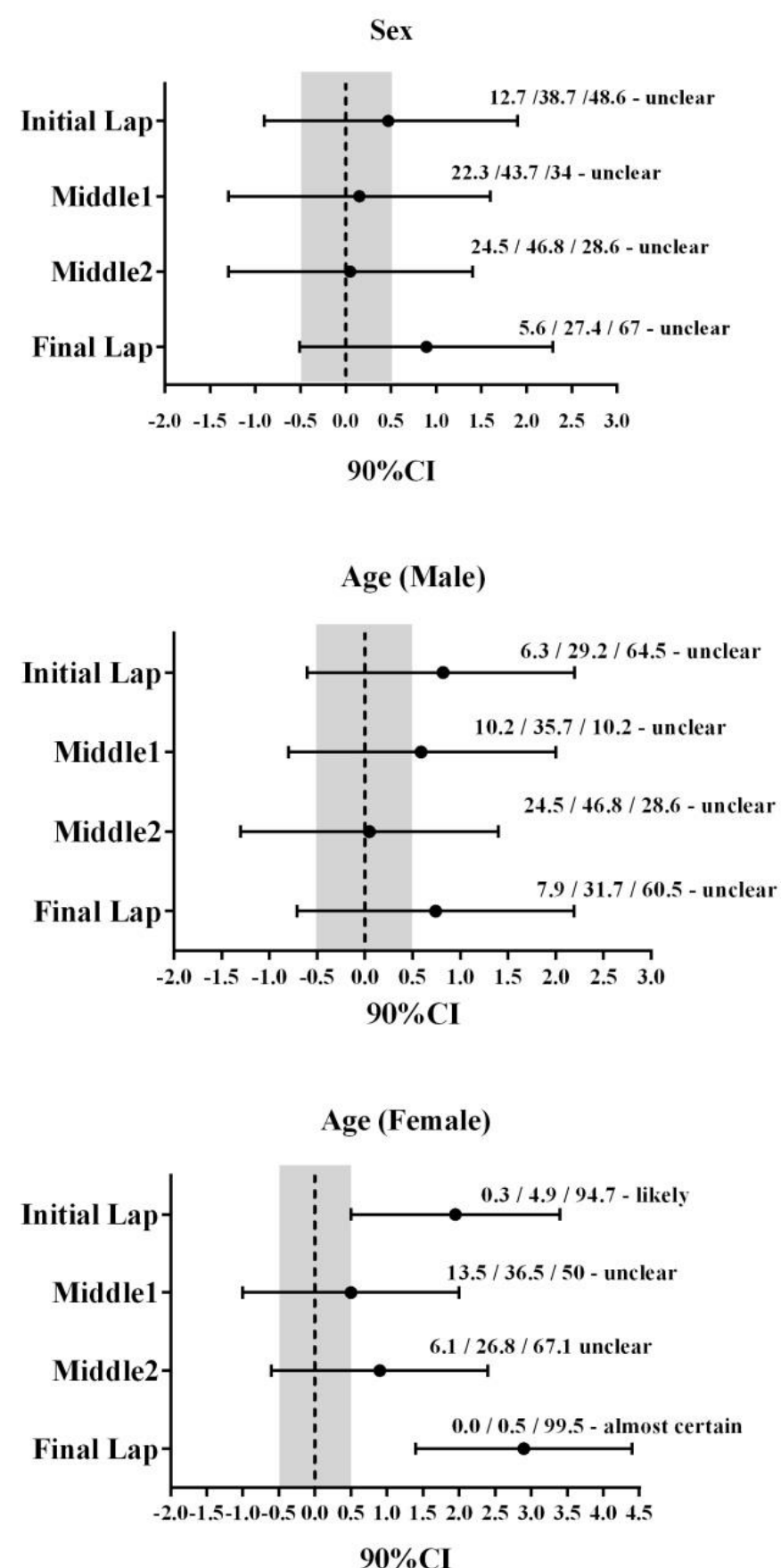

Figure 4. Magnitude-based inference for relative speed by race segments for sex and age. It is represented $90 \% \mathrm{CI}$ and quantitative chances $(\%)$.

\section{Discussion}

The present study aimed to characterize pacing strategy during an entire competitive season of XCO-MTB and to verify if performance level, sex and age would have some influence on the strategy adopted. Our main result was that the pattern of pacing strategy used during a season is characterized by a fast-start followed by speed maintenance independently of performance level, sex or age. Regarding performance level, in Elite categories ( $\mathrm{M}$ and $\mathrm{F})$, but not $\mathrm{U} 23$ ( $\mathrm{M}$ and $\mathrm{F}$ ), high performance athletes performed the final lap in higher relative speeds than low performance ones. Finally, the age influence the relative speed distribution in female athletes, but not in male ones.

Pacing strategy has been studied in different sports and events (Abbiss and Laursen, 2008; Renfree, Carmo, and Martin, 2016; Renfree, West, Corbett, Rhoden, and St Clair Gibson, 2012). Specifically in cycling, most of the studies used a wellcontrolled laboratory set-up or timetrials in flat courses and the results cannot be extrapolated to a real XCOMTB race, which is performed in courses with topography variation and with a high demand of technical skills (Impellizzeri and Marcora, 2007). In one of the few studies with XCO-MTB, Abbiss et al. (2013) observed a constant pacing strategy with the initial lap slower than the second lap in XCO-MTB World Championships race. On the other hand, in the present study we observed a well characterized faststart strategy during a UCI entire season. The discrepant results could be influenced by terrain characteristics. While Abbiss et al. (2013) studied only one race in a specific circuit, we analyzed the pacing in 6 World Cup races and 1 World Championship race. Therefore, a more global analysis involving different races could have contributed to dissipate the influence of technical characteristics of the terrain or a tactic decision in the initial part of one determined 
race on pacing strategy. In fact, our results corroborated with more recently studies during simulated (Viana, Pires, Inoue, and Santos, 2018) and real (Granier et al., 2018) XCO-MTB race which indicated a fast-start pacing strategy followed by an even pace.

The use of a fast-start strategy seems to be more useful and tactical decision for XCO-МТВ athletes, given in the initial part of the race they need a good position in the peloton to have some advantage during the narrower parts of the circuit, where overtake is difficult (Impellizzeri and Marcora, 2007). Macdermid and Morton (2012) observed a positive association between the start position and the final position, suggesting the importance of a fast-start pacing strategy. In addition, the observed fast-start strategy could also be explained by the influence of the "herd behavior" in a decision-making process (Renfree et al., 2015) since Koning et al. (2016) showed that the presence of a virtual opponent changed the pacing strategy during a 4-km time trial cycling race where a faster initial 400-m was observed when a virtual opponent performing a fast-start was introduced in the race. Thus, we can speculate that the fast-start strategy observed in XCO-MTB race is a decision-making process influenced by tactical and a herd behavior aspects.

After the fast-start we observed small variation in relative speed until the final lap. These results are similar to the findings of Bossi et al. (2017), where most cycle-cross cyclists did not vary their speed between laps by more than 5\% around the average race speed. In addition, similar results were also observed by Martin et al. (2012) during a XCO-MTB race of $\sim 1.5 \mathrm{~h}$ in 6 recreationally trained cyclists where an even pacing strategy was used across the 5 laps. Therefore, our data confirm the previous one and suggest that during XCO-MTB races athletes usually maintain an even-pace after the initial lap.

However, when we analyzed the pacing strategy by performance level, it was observed that in both Elite categories ( $\mathrm{M}$ and $\mathrm{F}$ ) high performance athletes performed the final lap in higher relative speeds ( $99 \%$ of average speed) than low performance ones ( $\sim 97 \%$ of average speed). Similar results were found in runners that in spite of similar initial speeds, athletes with poor performance were not able to maintain their speeds than athletes with better performance (Esteve-Lanao et al., 2014; Renfree and St Clair Gibson, 2013).

Viana, Pires, Inoue, and Santos (2018) showed that when athletes adopted a fast-start pacing strategy in a simulated XCO-MTB race there was a greater psychophysiological stress during the initial phase, as suggested by a higher heart rate and rating of perceived exertion in first lap. (Hays, Devys, Bertin, Marquet, and Brisswalter, 2018) observed that during a XCO-MTB race the contributions of aerobic and anaerobic metabolic pathways vary between laps. The first lap was associated with a significant decrease in blood $\mathrm{pH}$ and $\mathrm{HCO} 3$ as well as a significant increase in blood lactate levels, which reflect high solicitation of the anaerobic pathway. These variables tended to reduced following laps 2 and 3 , but still remained higher than pre-exercise values. Therefore, the fast-start can be risky, especially for low performance athletes, due the great physiological disturbance, which could impair the rest of the race and consequently the overall performance (de Koning et al., 2011; Tucker, 2009). In fact, the ability to maintain the speed during the last lap seems to be associated with athletes' physiological characteristics. Carmo et al. (2015) showed in runners the relationship between physiological characteristics and the ability to maintain the speed after an enforced fast-start. Athletes with higher peak speed during an incremental test and higher speed associated with maximal oxygen consumption were able to improve their performance after an enforced fast-start during a $10-\mathrm{km}$ running race. Future studies should be conducted to understand what and how these physiological variables could influence on pacing and performance level in a XCO-MTB race. 
Although differences in pacing strategy have been suggested to be influence by sex during a marathon (March, Vanderburgh, Titlebaum and Hoops, 2011), we did not observe differences in relative speed distribution between male and female athletes during a XCO-MTB race. On the other hand, Abbiss et al. (2013) showed a more variable pacing strategy in female athletes and attributed than to a more time spent during technical up hills and down hills. Given the technical characteristics of the circuit seems to directly influence the pacing, when we analyzed seven different races these technical influence on pacing strategy could be diluted. Therefore, we can suggest that during a XCO-MTB season the relative speed distribution pattern is similar for both male and female athletes. However, female athletes could be more susceptible to terrain characteristics.

When we analyzed age-group we did not observe differences between EliteM and U23M. However, different speed distribution between EliteF and U23F was observed. The U23F athletes started the race in higher relative speed than EliteF, but they were not able to maintain it during the race and finished with significantly lower relative speed. The differences related to age-group between male and female could be related to the lower absolute and relative power in women, which seems to impair the acceleration and transposition of obstacle when compared to male athletes (Impellizzeri et al., 2008) and this response could be more exacerbated when EliteF is compered to U23F. On the other way, this difference could be related to athlete's experience. Micklewright, Papadopoulou, Swart and Noakes (2010) observed that the pacing strategy in a $20-\mathrm{km}$ time-trial was defined based on the interaction between race distance and previous experiences. For instance, Swart et al. (2009) observed that as the 40-km time-trials were repeated, the athletes improved both their strategy and performance, which seems to be associated to a better decision making on the strategy used. In fact, differences in pacing strategy between young endurance athletes has been attributed to experience and decision-making process (Elferink-Gemser and
Hettinga, 2017). Thus, it is reasonable to assume that, despite competing at a high level, U23F athletes could have lower absolute and relative power and be less experience than Elite athletes, which seems influence on the decision making process and pacing strategy during the race. However, more data are necessary to support this information.

A limitation of the present study was that the data was accessed throughout the public domain website without more controlled power output, cadence or HR values. In the same way, the races involved different characteristics as altitude, weather conditions and technical difficulties which were not evaluated. Therefore, further investigations are necessary to evaluate performance level, sex or age influence according the race circuit characteristics since our data permitted to draw an overall presentation of pacing strategy in a XCO-MTB season.

In conclusion, during a competitive season of XCO-MTB the strategy used presents fast-start and subsequent maintenance of speed. In addition, better cross-country mountain bike cyclists (EliteM and EliteF) are able to maintain higher speeds in the final part of the race (final lap) compared to the lower placed finishers. Under 23 female athletes used to do a high faststart, but they are not able to sustain it in the final lap when compared to the Elite female athletes.

These results may help athletes and coaches to understand the pacing strategy used in an entire XCO-MTB session to development of training plans and more effective strategies for the races. For instance, since the athletes need to perform a fast-start but in the same time be able to maintain higher speeds during final lap the training program should involve sessions of high-intensity repeat sprint to improve the ability of athlete to produce high power output even in a metabolically unfavorable condition. Changes in pacing strategy can also be defined according the relative speed distribution and physiological characteristics of athletes. The 
same could be applied to young athletes, specially female or less experiences, who could try to maintain a lower relative speed variation during the race.

\section{Conflict of interest}

The author(s) declared no potential conflicts of interest with respect to the research, authorship, and/or publication of this article.

\section{References}

1. Abbiss, C. R., and Laursen, P. B. (2008). Describing and understanding pacing strategies during athletic competition. Sports Medicine, 38(3), 239-252

2. Abbiss, C. R., Ross, M. L., Garvican, L. A., Ross, N., Pottgiesser, T., Gregory, J., and Martin, D. T. (2013). The distribution of pace adopted by cyclists during a cross-country mountain bike World Championships. Journal of sports sciences, 31(7), 787-794

3. Bossi, A. H., O'Grady, C., Ebreo, R., Passfield, L., and Hopker, J. G. (2018). Pacing Strategy and Tactical Positioning During Cyclo-Cross Races. International journal of sports physiology and performance, 1-7. doi: 10.1123/ijspp.2017-0183

4. Cangley, P., Passfield, L., Carter, H., and Bailey, M. (2011). The effect of variable gradients on pacing in cycling time-trials. International Journal of Sports Medicine, 32(2), 132-136.

5. Costa, V. P., and de OLIVEIRA, F. R. (2010). Heart rate response during mountain bike crosscountry races. Revista brasileira de Educação Física e Esporte, 24(3), 379-387

6. de Koning, J. J., Foster, C., Bakkum, A., Kloppenburg, S., Thiel, C., Joseph, T., . . Porcari, J. P. (2011). Regulation of pacing strategy during athletic competition. PLoS One, 6(1), e15863.

7. Deaner, R. O., and Lowen, A. (2016). Males and Females Pace Differently in High School CrossCountry Races. The Journal of Strength \& Conditioning Research, 30(11), 2991-2997.

8. do Carmo, E., Barroso, R., Renfree, A., Gil, S., and Tricoli, V. (2015). Influence of an Enforced FastStart on 10-km Running Performance. International journal of sports physiology and performance, 11(6):736-741.
9. Elferink-Gemser, M. T., and Hettinga, F. J. (2017). Pacing and Self-regulation: Important Skills for Talent Development in Endurance Sports. International journal of sports physiology and performance, 12(6), 831-835.

10. Esteve-Lanao, J., Larumbe-Zabala, E., Dabab, A., Alcocer-Gamboa, A., and Ahumada, F. (2014). Running World Cross-Country Championships: a unique model for pacing. International journal of sports physiology and performance, 9, 10001005.

11. Granier, C., Abbiss, C. R., Aubry, A., Vauchez, Y., Dorel, S., Hausswirth, C., and Le Meur, Y. (2018). Power Output and Pacing During International Cross-Country Mountain Bike Cycling. International journal of sports physiology and performance, 13(9), 1243-1249.

12. Hays, A., Devys, S., Bertin, D., Marquet, L. A., and Brisswalter, J. (2018). Understanding the Physiological Requirements of the Mountain Bike Cross-Country Olympic Race Format. Frontiers in Physiology, 9, 1062.

13. Hopkins, W., Marshall, S., Batterham, A., and Hanin, J. (2009). Progressive statistics for studies in sports medicine and exercise science. Medicine \& Science in Sports \& Exercise, 41(1), 3.

14. Impellizzeri, F., Ebert, T., Sassi, A., Menaspa, P., Rampinini, E., and Martin, D. (2008). Level ground and uphill cycling ability in elite female mountain bikers and road cyclists. European journal of applied physiology, 102(3), 335-341

15. Impellizzeri, F., Sassi, A., Rodriguez-Alonso, M., Mognoni, P., and Marcora, S. (2002). Exercise intensity during off-road cycling competitions. Medicine and science in sports and exercise, 34(11), 1808-1813

16. Impellizzeri, F. M., and Marcora, S. M. (2007). The physiology of mountain biking. Sports Medicine, 37(1), 59-71

17. Konings, M. J., Schoenmakers, P. P., Walker, A. J., and Hettinga, F. J. (2016). The behavior of an opponent alters pacing decisions in 4-km cycling time trials. Physiology \& behavior, 158, 1-5

18. Macdermid, P. W., and Morton, R. H. (2012). A longitudinal analysis of start position and the outcome of World Cup cross-country mountain bike racing. Journal of sports sciences, 30(2), 175182 
19. March, D. S., Vanderburgh, P. M., Titlebaum, P. J., and Hoops, M. L. (2011). Age, sex, and finish time as determinants of pacing in the marathon. Journal of Strength \& Conditioning Research, 25(2), 386-391

20. Martin, L., Lambeth-Mansell, A., BerettaAzevedo, L., Holmes, L. A., Wright, R., and Gibson, A. S. C. (2012). Even between-lap pacing despite high within-lap variation during mountain biking. International journal of sports physiology and performance, 7(3), 261-270

21. Micklewright, D., Papadopoulou, E., Swart, J., and Noakes, T. (2010). Previous experience influences pacing during $20 \mathrm{~km}$ time trial cycling. British journal of sports medicine, 44(13), 952-960

22. Micklewright, D., Angus, C., Suddaby, J. S., St Clair Gibson, A., Sandercock, G., Chinnasamy, C. (2012). Pacing strategy in schoolchildren differs with age and cognitive development. Medicine and science in sports and exercise, 44, 362-369

23. Renfree, A., Crivoi do Carmo, E., and Martin, L. (2016). The influence of performance level, age and gender on pacing strategy during a $100-\mathrm{km}$ ultramarathon. European Journal of Sport Science, 16(4), 409-415

24. Renfree, A., Crivoi do Carmo, E., Martin, L., and Peters, D. M. (2015). The Influence of Collective Behavior on Pacing in Endurance Competitions. Frontiers in Physiology, 6, 373. doi: 10.3389 /fphys.2015.00373

25. Renfree, A., and St Clair Gibson, A. (2013). Influence of different performance levels on pacing strategy during the Women's World Championship marathon race. International journal of sports physiology and performance, $8(3), 279-285$

26. Renfree, A., West, J., Corbett, M., Rhoden, C., and St Clair Gibson, A. (2012). Complex interplay between determinants of pacing and performance during $20-\mathrm{km}$ cycle time trials. International journal of sports physiology and performance, $7(2), 121-129$

27. Swart, J., Lamberts, R. P., Lambert, M. I., Lambert, E. V., Woolrich, R. W., Johnston, S., and Noakes, T. D. (2009). Exercising with reserve: exercise regulation by perceived exertion in relation to duration of exercise and knowledge of endpoint. British journal of sports medicine, 43(10), 775-781
28. Tomazini, F., Pasqua, L. A., Damasceno, M. V., Silva-Cavalcante, M. D., de Oliveira, F. R., LimaSilva, A. E., and Bertuzzi, R. (2015). Head-to-head running race simulation alters pacing strategy, performance, and mood state. Physiology \& behavior, 149 , 39-44

29. Tucker, R. (2009). The anticipatory regulation of performance: the physiological basis for pacing strategies and the development of a perceptionbased model for exercise performance. British Journal of Sports Medicine, 43(6), 392-400

30. Viana, B. F., Pires, F. O., Inoue, A., and Santos, T. M. (2018). Pacing Strategy During Simulated Mountain Bike Racing. International journal of sports physiology and performance, 13(2), 208213 